\title{
Combined microsurgical PICA-PICA bypass and endovascular parent artery occlusion for a ruptured dissecting vertebral artery aneurysm
}

Robert M. Starke M.D., M.Sc., ${ }^{1}$ Dale Ding M.D., ${ }^{1}$ Christopher R. Durst, M.D., ${ }^{2}$ R. Webster Crowley, M.D. ${ }^{1,2}$ and Kenneth C. Liu, M.D.1,2

University of Virginia, Departments of Neurological Surgery ${ }^{1}$ and Radiology and Medical Imaging ${ }^{2}$, Charlottesville, Virginia

Dissecting vertebral artery (VA) aneurysms are difficult to obliterate when the parent artery cannot be safely occluded. In this video, we demonstrate a combined microsurgical and endovascular treatment technique for a ruptured, dissecting VA aneurysm incorporating the origin of the posterior inferior cerebellar artery (PICA). We first performed a PICA-PICA side-to-side bypass to preserve flow through the right PICA. An endovascular approach was then utilized to embolize the proximal portion of the aneurysm from the right VA and the distal portion of the aneurysm from the left VA.

The video can be found here: http://youtu.be/dkkKsX2BiJl.

KEY WORDS bypass; endovascular procedures; intracranial aneurysm; parent artery occlusion; stroke; subarachnoid hemorrhage 